\title{
Fuzzy Epidemiology: Measures for Observational Epidemiological Studies
}

\section{CURRENT STATUS: POSTED}

Research Square

Ana Cláudia Oliveira Melo

Universidade Federal da Paraiba Centro de Ciencias da Saude

anaclaudiaemelo@gmail.comCorresponding Author

ORCiD: https://orcid.org/0000-0003-0856-1972

Laisa Ribeiro de Sá

Universidade Federal da Paraiba

Rodrigo Pinheiro de Toledo Vianna

Universidade Federal da Paraiba

Ronei Marcos de Moraes

Universidade Federal da Paraiba

DOI:

10.21203/rs.2.19661/v1

SUBJECT AREAS

Statistical Epidemiology

KEYWORDS

Epidemiological Frequency Measures, Risk analysis measures, Fuzzy Set Theory, Possibilities theory 
Abstract

Background Epidemiological studies bring forth classic epidemiological measures calculation that are based on resulting quantities of dichotomic categorization of individuals, such as in events: diseased, non-diseased, exposed or unexposed. Dichotomic categorizations discard inherent uncertainties and subjectivities from the illness process the exposure which generate information losses on the measures. The fuzzy set theory categorizes each individual, allowing the soft transit amongst the events and considering the uncertainties and subjectivities. For the calculation of these measures, the fuzzy possibility theory is useful. Although there is already the proposition of making use of this methodology to the calculation of association and risk measures, there are no additional studies, in the literature, that characterize or apply the measures in epidemiological studies. Neither there are proposed calculations of other epidemiological measures or studies explaining the contribution of the resulting epidemiological measure of this methodology. This paper aims to increase the epidemiological measures sets to observational studies, using fuzzy set and possibility theories in the calculation of the denominated fuzzy epidemiological measures, featuring them in an original way. Methodology The proposed fuzzy measures were based in classic epidemiological measures. An observational study was simulated on a case-control type and fuzzy theories on the categorization of the individuals and for the calculation of fuzzy measures were applied. The simulations and calculations were performed by the software $\mathrm{R}$.

Results It was graphically observed the incorporation of uncertainties and subjectivities in the study population categorization. Comparing the classic to the fuzzy measures, it was observed that the contribution of the embedded uncertainties and subjectivities on the fuzzy measure presented a more complete final information about the illness process and exposure of the individuals. The graphic behavior of the proposed measures and of the already existent ones were characterized.

Conclusion The fuzzy set epidemiological measures changes the paradigm of measures restricted to one numerical value. The information of the new fuzzy measures is seen as more trustworthy and helpful to decision making health managers, regarding which policies must be considered in accordance to the susceptible of harm and exposure in every population of each case scenario. 
Full Text

Due to technical limitations, full-text HTML conversion of this manuscript could not be completed. However, the manuscript can be downloaded and accessed as a PDF.

Figures
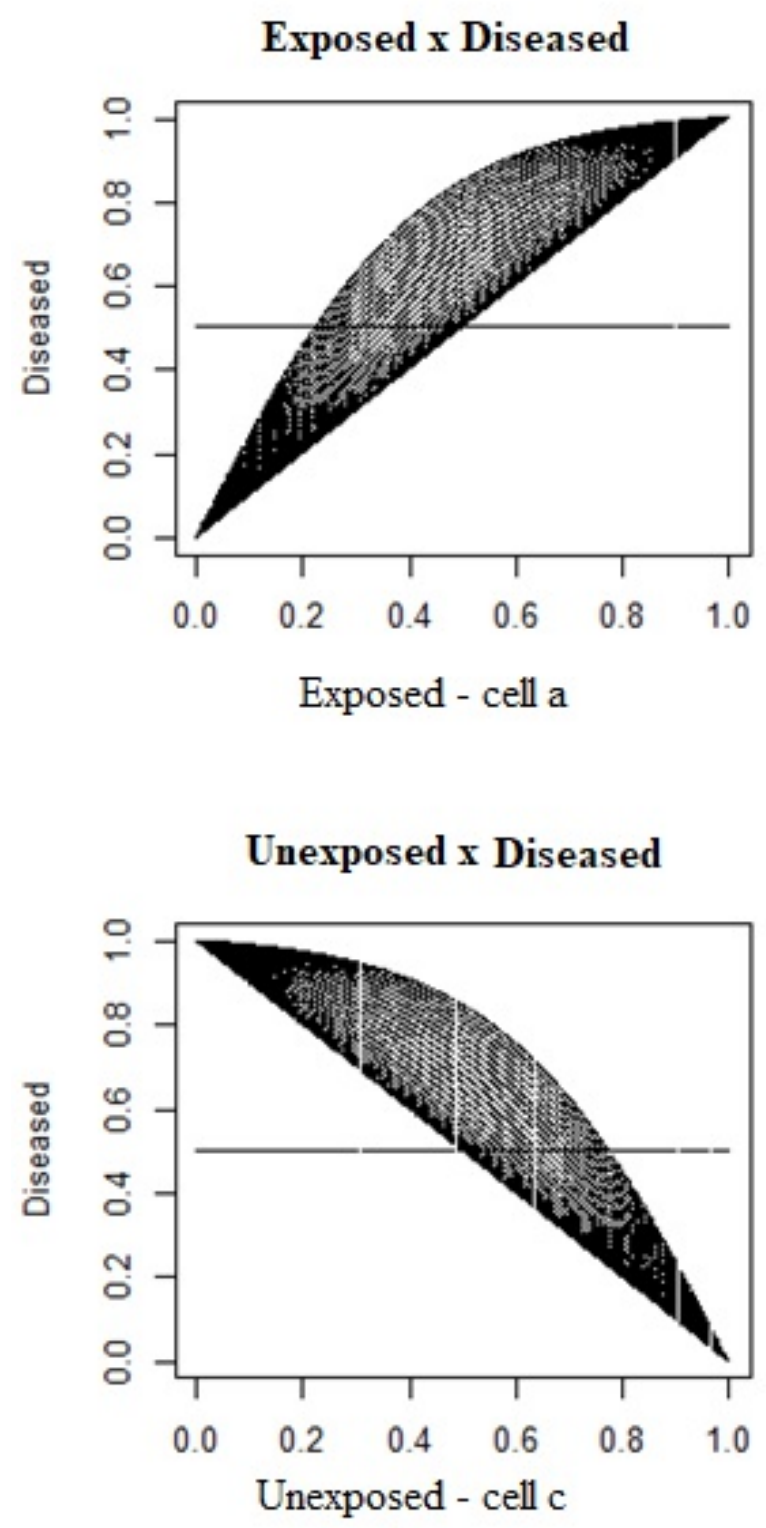
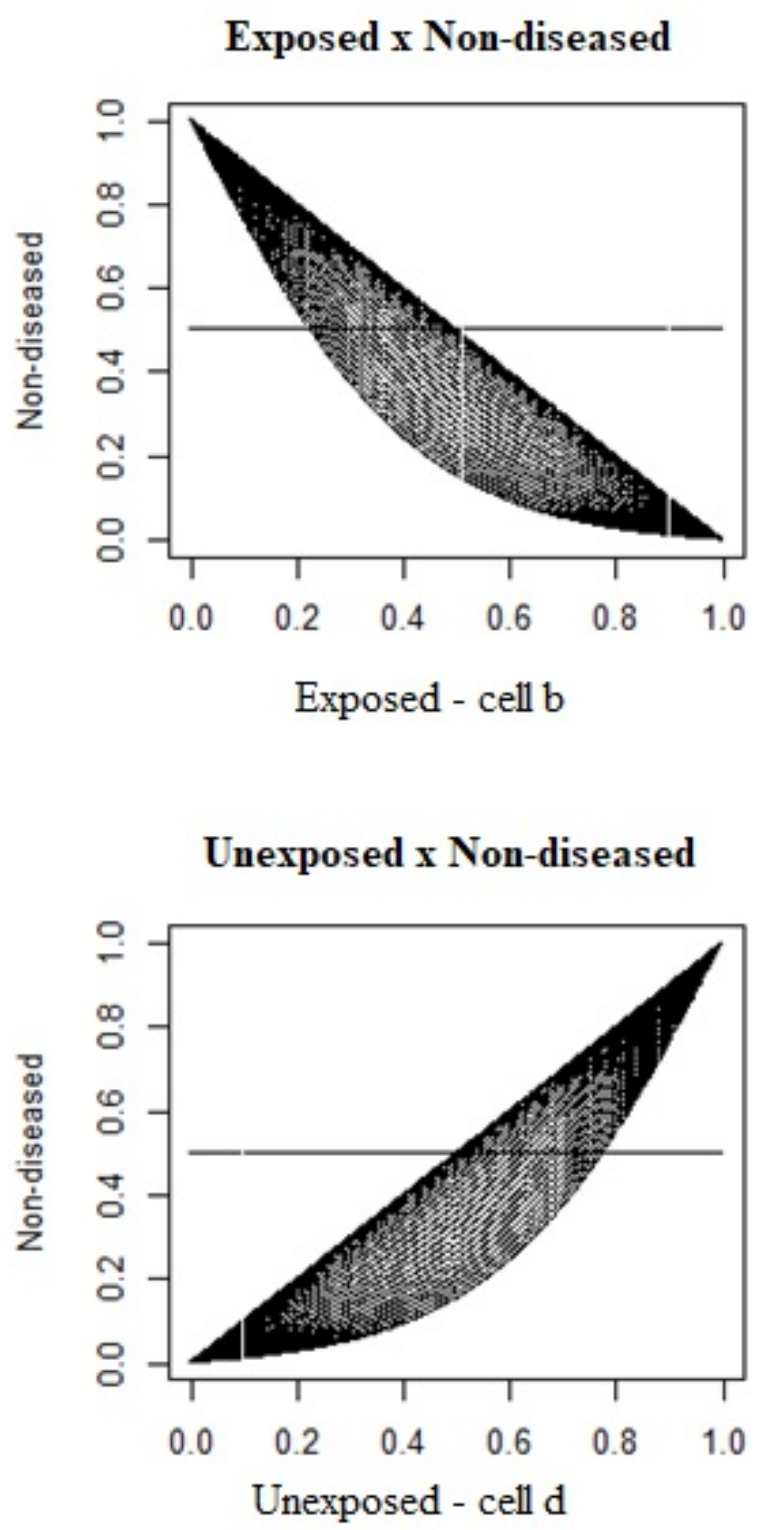

Figure 1

Mass graph of individuals classified according to their membership functions $\mu(x)$ under the aspects of the study $\left(E, E, D, D^{-}\right)$. 

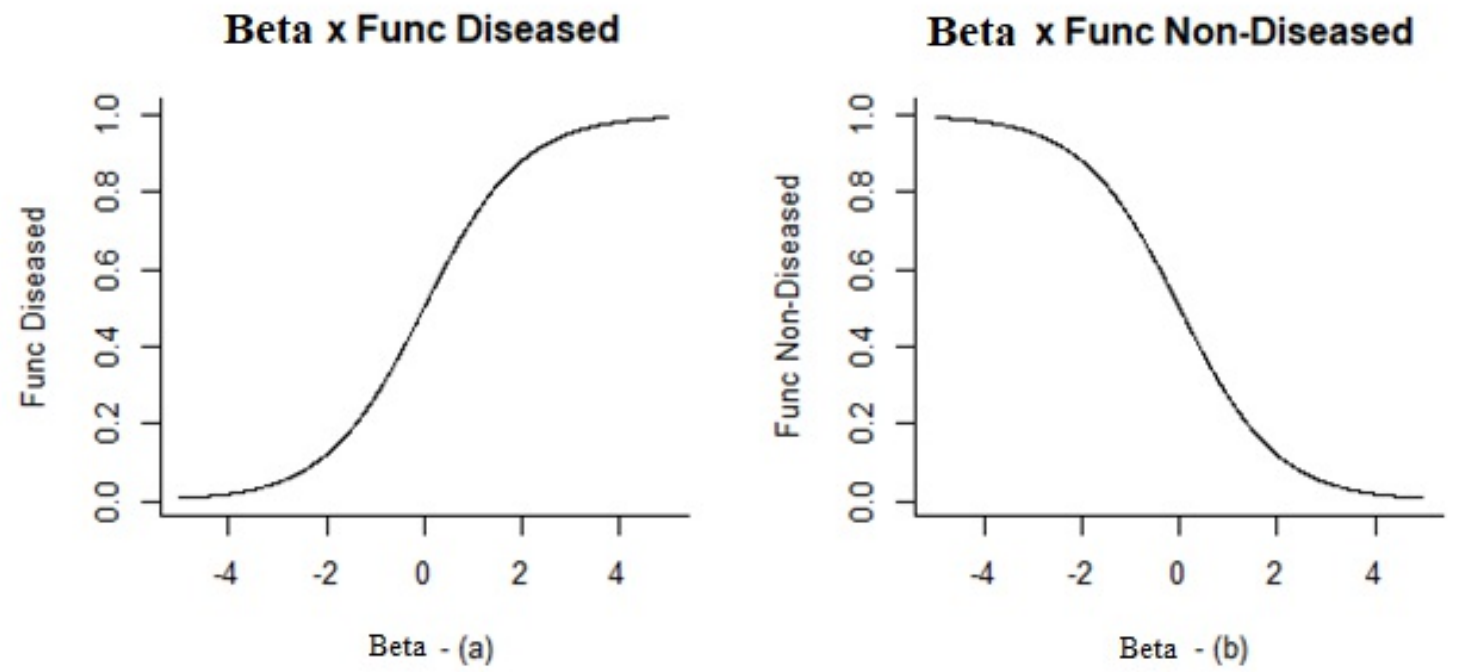

Figure 2

Behavior graphs of the diseased $\mu_{-} D(x)$ (graph a) and non-diseased $\mu_{-} D^{-}(x)$ (graph $\left.b\right)$ membership functions according to the intensity of the Odds Ratio of falling ill $(\beta)$.

Func Exposed x FP

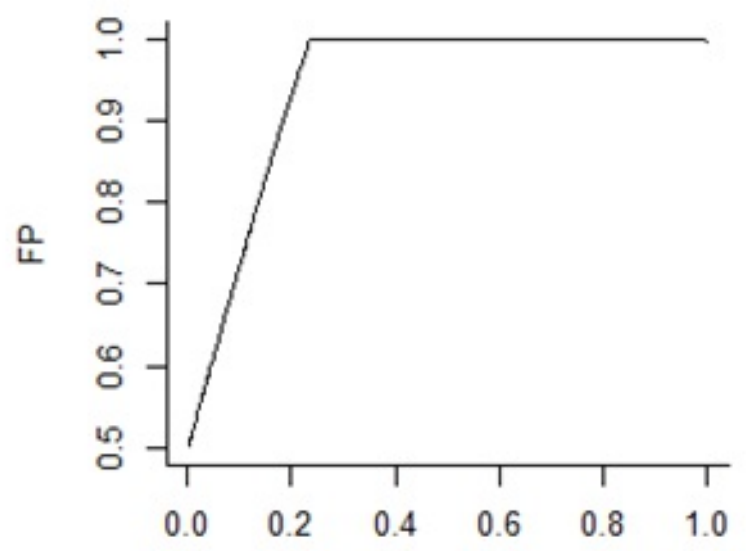

Func Exposed - (a)
Beta $\times$ FP

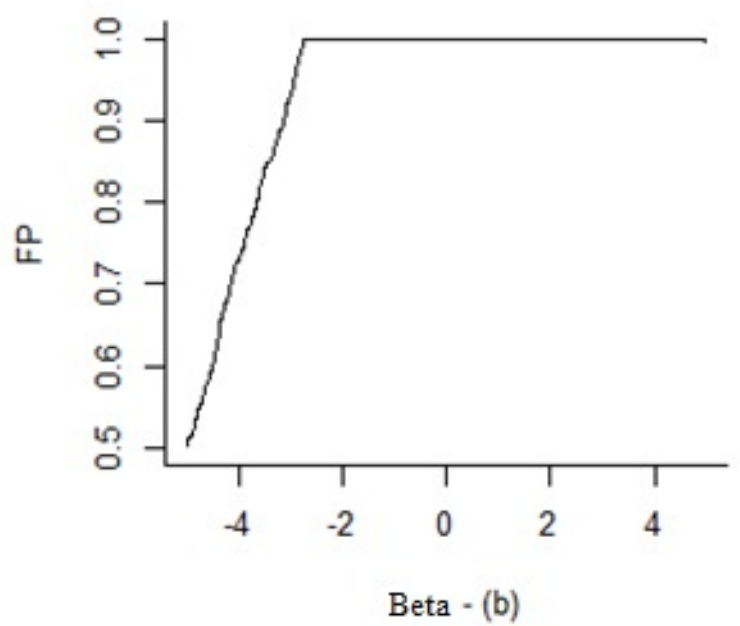

Figure 3

Behavior graphs of the FP, according to the exposed membership function $\mu_{-} E(x)$ (graph a) and the intensity of the Odds ratio of falling ill $(\beta)$ (graph b). 
Func Exposed x FIAE

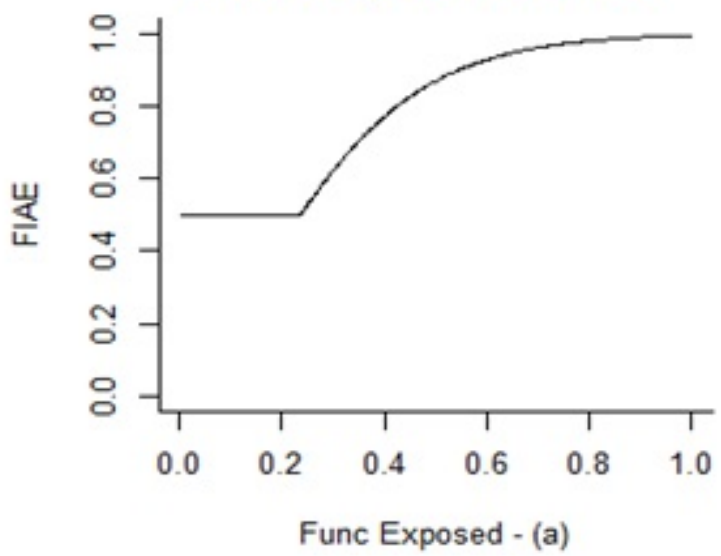

Beta $x$ FIAE

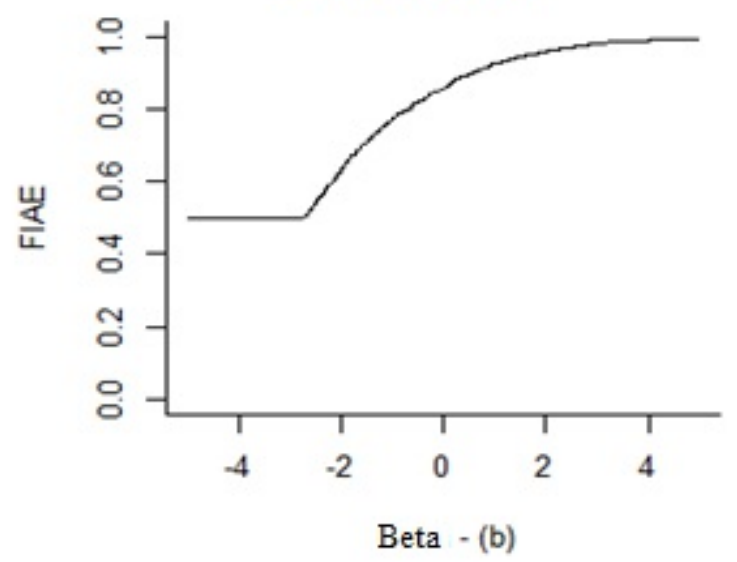

Figure 4

Behavior graphs of the FI_AE, according to the exposed membership function $\mu_{-} E(x)$ and the intensity of the Odds ratio of falling ill $(\beta)$.

Func Exposed x FOE

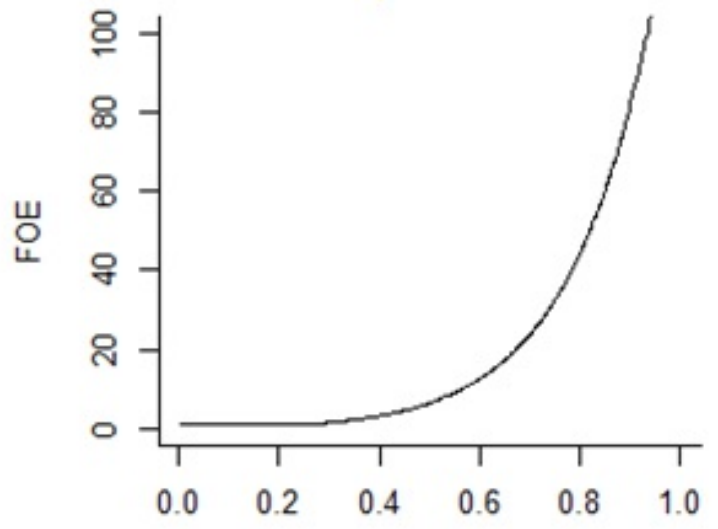

Func Exposed - (a)
Beta $x$ FOE

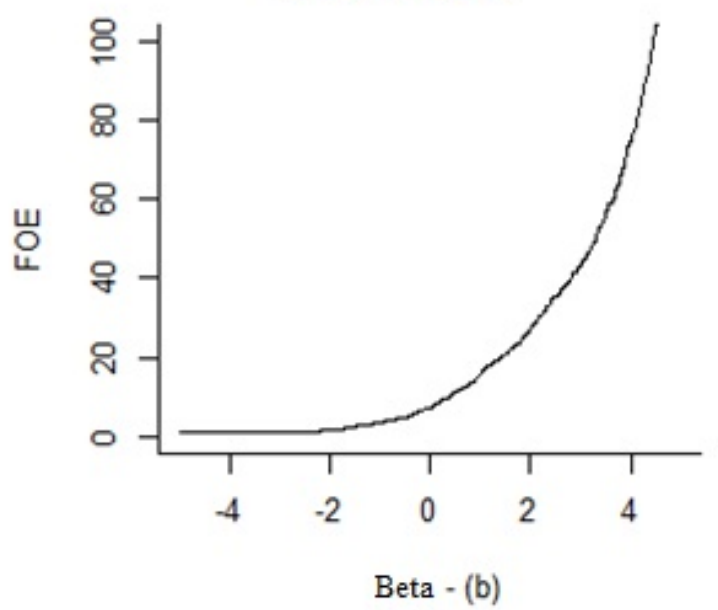

Figure 5

Behavior graphs of the $\square \mathrm{FO} \square_{-} E$, according to the exposed membership function $\square(\mu \square[E(x))$ (graph a) and the intensity of the odds ratio of becoming ill ( $\beta$ ) (graph b). 
Beta $x$ FOR

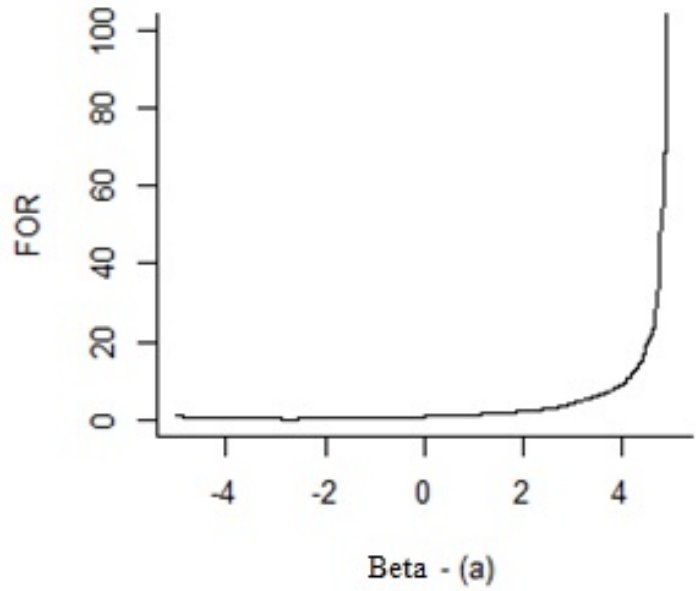

Beta $x$ FRR

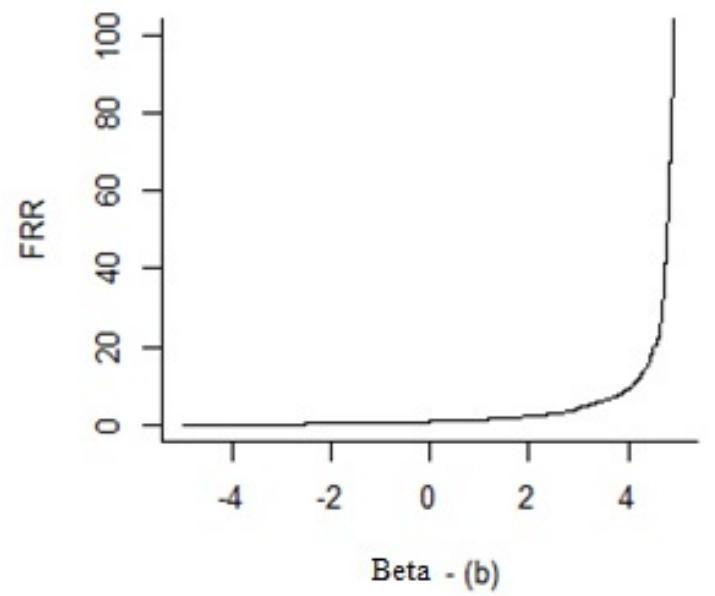

Figure 6

Behavior graphs of FOR and FRR for simulated values of the beta parameter. 